\title{
LA FUNCIÓN DE LA LÓGICA CLÁSICA EN LA LLAMADA "LÓGICA CUÁNTICA"*
}

CARLOS LUNGarzo

Universidade Estadual de Campinas, Brasil

El objeto de este trabajo es el estudio del papel desempeñado por las técnicas lógicas de carácter clásico en la construcción de ciertas estructuras llamadas (ambiguamente) "lógicas cuánticas". Las presuposiciones lógicas sobre las cuales nos basamos son de carácter extremadamente elemental, y todas ellas están contenidas, por ejemplo, en el libro de Shoenfield [18]. A su vez, los conceptos estrictamente físicos pueden verse en los primeros capítulos de cualquier texto introductorio de mecánica cuántica, como, por ejemplo, Merzbacher [14]. En cambio, una idea relativamente precisa de problemas de epistemología de la física puede ser muy útil. La referencia canónica para este tema incluye Bunge [3], [4] y [5]. Dicho sea de paso, la coincidencia entre algunas tesis aquí defendidas y las que Bunge propone en esos y otros trabajos, no debe sorprender. Sin embargo, cualquier polémica de carácter metafísico, como la plausibilidad o implausibilidad del realismo en mecánica cuántica, queda totalmente fuera del espíritu de este artículo, que es esencialmente lógico.

\section{El enfoque ortodoxo}

Los autores a quienes se deben las primeras formulaciones sistemáticas de la lógica cuántica, son, curiosamente, los primeros en objetar la plausibilidad del término "lógica", cuando se lo aplica a los retículos de las proposiciones cuánticas.

" En todo este texto, la palabra "cuántico" y sus derivados, son usados en el sentido de "cuántico no relativista". 
En efecto, Jauch [10], p. 77, considera que el uso del vocablo "quantum logic in contradistinction to ordinary logic", ha causado gran confusión. Pero, si bien su manera de aclararla consiste en advertir que la "lógica" cuántica pretende la formalización de hechos y la lógica formal (ordinaria), el análisis del significado de proposiciones, sin embargo, su crítica no se profundiza y el balance final de la misma parece dejar la opinión de que ambos universos de discurso son muy diferentes.

Por su parte, Piron [15], p. 441, hace observaciones mucho más precisas: "En fait, ces axiomas (los de las estructuras cuánticas) ne sont que des régles de calcul et la logique habituelle s'applique sans avoir besoin d'être modifiée."

Las pruebas dadas por el autor en la época en que redactó su tesis, parecen contundentes. En particular, una de las objeciones gira en torno de la ausencia de implicación, en la lógica cuántica, ya que nociones tales como "implicación" o, a veces, "deducción", parecen básicas en cualquier tipo de lógica, y el condicional resulta quizá la única conectiva que ningún cálculo lógico puede dejar de tener.

Sin embargo, en la "lógica" cuántica, tal como fue construida y analizada por Birkhoff, von Neumann, Jauch, Piron y otros, no existe ninguna operación lógica equivalente a un condicional. La relación de orden entre las proposiciones, que tiene propiedades formales análogas a las de la implicación, no es una operación dentro del retículo, ya que si, por ejemplo, $x$ e $y$ son dos proposiciones cuánticas, la afirmación de que " $x$ percede a $y$ " en el orden, simbolizada por:

$$
x \leqslant y
$$

no tiene por qué ser una proposición cuántica. Más aún, ella es simplemente una aserción de la teoría en la cual las estructuras cuánticas son descritas, y carece directamente de sentido considerarla como el resultado de una operación proposicional, como muestra el ejemplo:

$$
(x \leqslant y) \leqslant z \text {. }
$$


Esta objeción, empero, no invalidaría a nuevas estructuras construidas de manera totalmente análoga, pero que tuvieran una operación (en sentido estricto), cuyas propiedades la asemejaran razonablemente a una implicación. Eso fue efectivamente lo que sucedió con la introducción de una nueva operación en los retículos ortomodulares, tal como se hace, por ejemplo, en el trabajo de Hardegree [9], p. 1352 y ss. De hecho, este autor introduce un cierto número de operaciones que satisfacen ciertos criterios mínimos que, en su opinión, serían suficientes para caracterizar a las mismas como implicaciones: (i) la propiedad de modus ponens, y (ii) la propiedad de que si $x$ precede a $y$ en el orden del retículo, entonces el elemento que resulta de aplicar la operación al par $(x, y)$ es igual a la unidad del retículo. Pese a que la propiedad transitiva falle, y, según reconoce el autor, eso llevaría a rechazar la legitimidad de las operaciones como posibles implicaciones, es indudable que este argumento rebate parcialmente las objeciones de Piron.

Creemos que deben buscarse razones más fuertes, de carácter metodológico, para objetar la afirmación de que las estructuras cuánticas constituyen una lógica. Por empezar, consideremos tres posibles niveles en los cuales podrían aparecer los elementos de la teoría actualmente llamada "lógica cuántica":

(1) La "lógica" cuántica podría ser considerada como un conjunto de axiomas del cálculo proposicional (o sea, estrictamente lógicos), con modus ponens, por ejemplo, como regla de inferencia. De esa manera, las estructuras cuánticas conocidas (impropiamente llamadas "lógicas cuánticas") serían las álgebras abstractas asociadas con ese cálculo (en el sentido corriente de "álgebra asociada" que se utiliza en la lógica algebraica, p. ej., en Raziowa [17], p. 181).

Este tipo de construcción es perfectamente posible. La "logización" de las estructuras ortomodulares cuánticas puede hacerse de manera análoga a la "logización" de los retículos modulares de Birkhoff-von Neumann, hecha por Kotas [12], 
p. 32. Inclusive, esta lógica tiene todas las características formales de una "auténtica" lógica, ya que por ejemplo, es posible dar una formulación de ella con axiomas esquemas, tal como el mismo Kotas [13] hizo posteriormente.

En este sentido, la lógica cuántica sería una de las múltiples familias de cálculos proposicionales no clásicos, como lo es la intuicionista, la implicativa, la mínima, la modal, etc. En el mejor de los casos, lo que ella ofrecería sería de interés exclusivo para los lógicos no clásicos.

(2) Podemos pensar también una "lógica" cuántica a nivel de teoría basada en la lógica clásica de predicados. En efecto, consideremos las versiones usuales de esta lógica provista de igualdad, y agreguemos los axiomas de una teoría de conjuntos. Nuestro lenguaje específico ("lenguaje para la teoría cuántica") podría ser el siguiente:

$$
\mathrm{L}(\mathrm{TC})=\left\{\mathrm{v}, \wedge,^{\prime}, 0,1\right\}
$$

Luego, agregamos a los axiomas de la lógica subyacente provista de los postulados de la teoría de conjuntos, las proposiciones que establecen:

(i) orden parcial completo

(ii) propiedades de acotación de 0 y 1 , o sea:

$(x)(x$ ) $1=1)$

$(x)(x \wedge 0=0)$

(iii) propiedades usuales de ortocomplementación

(iv) propiedad de ortomodularidad

(v) propiedades de atomicidad, o sea:

(v.1) Todo elemento domina algún átomo

(v.2) Los intervalos abiertos cuyo "diámetro" es un átomo, son vacíos.

Este esquema, que es adecuado para la formulación ortodoxa de Jauch [10], cap. v, y Piron (donde todavía se deben agregar las condiciones sobre el conjunto de estados), es válida, con las obvias modificaciones, para las teorías de Gudder [7] y [8], Pulmannova [16] y Deliyannis, entre 
otras muchas. Las diferencias técnicas entre ellas son totalmente irrelevantes desde el punto de vista de nuestra argumentación.

(3) Finalmente, en vez de considerar las teorías, podemos llamar "lógicas" cuánticas, a las estructuras que son modelos de esas teorías. En la versión ortodoxa, esas estructuras serían los modelos:

$$
(\mathrm{L}, \mathrm{v}, \wedge, ', 0, \hat{\mathrm{i}})
$$

(para L cualquier conjunto no vacío) del sistema de postulados $(i)-(v)$. (Es obvio que los postulados de la teoría de conjuntos que podemos emplear para axiomatizar (i)-(v) son muy débiles, razón por la cual el modelo no necesita ser muy "extraño".)

Pues bien: el uso habitual del término "lógica cuántica" en la literatura lógica y epistemológica, se refiere exclusivamente a este nivel (3), con excepción de algunos casos particulares. Es decir que, lo que se considera como lógica es simplemente una estructura algebraica o, eventualmente, algebraico-topológica. Está claro entonces que la denominación de "lógica cuántica" no es más acertada de lo que sería, por ejemplo "lógica grupal", si consideráramos un cálculo proposicional con los axiomas:

$$
\begin{gathered}
p+(q+r)=(p+q)+r \\
p+F=p \\
p+p=F
\end{gathered}
$$

(donde $\mathrm{F}$ es la clase de equivalencia de las proposiciones contradictorias), por el simple hecho de que el álgebra asociada con este cálculo proposicional es un grupo para la operación + con la unidad $\mathrm{F}$.

Se podría establecer nuevamente la analogía lógico-algebraica, y reclamar el derecho de las estructuras cuánticas a entrar dentro del "folklore" lógico, debido, entre otras cosas, a que ellas se comportan como álgebras asociadas, de la misma manera en que las álgebras pseudo-booleanas se compor- 
tan en relación con la lógica intuicionista. A pesar de que esta observación es absolutamente plausible, ella no constituye un elemento de juicio a favor de que la "lógica cuántica sea el formalismo adecuado para el razonamiento usual en la mecánica cuántica". La única conclusión admisible sería la de que los retículos cuánticos constituyen una formalización adecuada de las operaciones empíricas de los procesos de formulación de preguntas (correspondientes a las alternativas si-no) y de preparación de estados, y de que, a su vez, la lógica cuántica (propiamente dicha) es una lógica no clásica (construida en virtud del empeño de los lógicos que se interesan por este tipo de teorías), inspirada en la estructura algebraica de los retículos cuánticos.

\section{Formulaciones especiales}

Las presentaciones más recientes de estructuras aptas para la formalización de las proposiciones cuánticas, son susceptibles de un análisis similar al realizado para la versión ortodoxa, relativa a retículos ortomodulares atómicos. Uno de los casos más ilustrativos es posiblemente el de Kochen y Specker [11].

Para estos autores, la estructura algebraica de los observables corresponde a un álgebra parcial (p. 180), donde la suma el producto están definidos sólo parcialmente (específicamente, para los pares $(a, b)$ que satisfacen la relación de conmensurabilidad). Pero su enfoque comienza enfatizando los aspectos algebraicos de las estructuras consideradas, y sólo posteriormente se introduce una lógica proposicional cuántica, como sistema axiomático que recupera las propiedades de las álgebras de Boole parciales.

En efecto, las álgebras parciales son estructuras de la forma: (A, $\S,+, ., \bullet, \tilde{\mathrm{i}})$ con la tipificación habitual: $A$ es un conjunto no vacío, $\S$ es un predicado binario, $+\mathrm{y}$. son funciones binarias, • es una ley de composición externa (producto por escalares, en este caso reales) e $\tilde{i}$ una función de grado cero (el elemento "máximo" del álgebra). To- 
das las propiedades son fácilmente formalizables con el uso de cuantificadores: por ejemplo, la relación $x \oint y$ ( $x$ es conmensurable con $y$ ) es simétrica y reflexiva; todo elemento es conmensurable con la unidad, i.e., $x \S \tilde{1}$, y otras condiciones análogas a los axiomas de splitting de la versión reticular.

El procedimiento para la "recuperación" de la lógica a partir del álgebra, es un típico método de la teoría de modelos. Se construye un cálculo proposicional con los conectivos de disyunción y negación, y se caracteriza la validez de una fórmula en una cierta estructura de álgebra parcial, si el correspondiente polinomio booleano toma el valor $\tilde{1}$ para.valuaciones adecuadamente definidas. En este caso, la lógica proposicional (cuántica) correspondiente, surge como una construcción natural asociada a la estructura, la cual, a su vez, aparece como una formalización de la noción empírica de "observable", pero no se trata, sin embargo, de una lógica destinada a reemplazar a la clásica en el desarrollo de la matemática de la mecánica cuántica. La axiomatización resultante (p. 186) tiene interés propio como un cálculo proposicional de carácter no clásico, pero su motivación central radica en el hecho de describir proposicionalmente las álgebras parciales.

El análisis puramente semántico de las relaciones cuánticas, tampoco puede considerarse como una tarea que pretenda introducir una lógica cuántica en sustitución de la lógica clásica. En su interesante formulación modal de las teorías cuánticas, van Fraassen [19], proporciona interpretaciones que, utilizando técnicas análogas a los modelos de Kripke, unifican conceptos que en las formulaciones anteriores aparecen relativamente dispersos. El mismo autor formulará $[20]$, p. 600 , lo que creemos es una de las observaciones más lúcidas (inclusive, más evidentes) sobre la naturaleza de estas "lógicas":

Pero desde nuestro punto de vista, una lógica de la mecánica cuántica es simplemente un in- 
tento de dar cuenta, de manera sistemática, de las relaciones semánticas entre los enunciados elementales de esa teoría. Y estas relaciones semánticas deben ser deducidas de la teoría cuántica -ése es el sentido en el cual esta lógica es una lógica cuántica. No se quiere decir que sea la base para una formalización de la teoría, o para unos nuevos Principia (i.e., Principia $M a$ thematica) de carácter no standard.

Espero poder proporcionar, en lo siguiente, nuevos argumentos en favor de esta tesis, que en cierta medida ya queda avalada por nuestro análisis de la versión ortodoxa.

\section{El punto de vista de la teoría de modelos}

¿Cuáles son las motivaciones para considerar, finalmente, todas estas estructuras, es decir, retículos ortomodulares, conjuntos ortomodulares, álgebras de Boole parciales, modelos especiales, etc.? La respuesta es muy vieja y conocida y se remonta a Birkhoff, von Neumann, Reichenbach y otros precursores de los estudios lógico-epistemológicos de la mecánica cuántica. Hagamos, sin embargo, una mínima revisión, no tanto para recordar cosas ya bien sabidas, sino simplemente para unificar terminología y conceptuación.

Las proposiciones con significado empírico que interesan en la teoría física, son, en general, reducibles a proposiciones de doble alternativa: si o no. Detectamos la presencia o ausencia de una cierta propiedad en un cierto experimento. Pero el físico pretende también medir, y por lo tanto se interesa por proposiciones que afirmen que determinados elementos del espacio de fase del sistema considerado, reciben ciertos valores reales a través de funciones determinadas. En mecánica clásica, esas proposiciones constituyen, desde el punto de vista de la estructura que formaliza las operaciones empíricas, un álgebra de Boole. La razón es simple: prácticamente no existen proposiciones incompatibles. (La 
incompatibilidad es tan pequeña con relación al error experimental que puede despreciarse.) Pero en mecánica cuántica no es así. Los socorridos ejemplos que aparecen siempre en los trabajos de epistemología de la mecánica cuántica muestran casos de proposiciones incompatibles (p.ej., "la partícula $x$ tiene en el momento $t$, una coordenada $q$ y un momento canónico p").

Luego, esas proposiciones deben ser dispuestas en una estructura que haga justicia a la existencia de esa incompatibilidad. El método de conceptualización allí utilizado es bien conocido:

(a) De los datos empíricos, a la formulación matemática más inmediata, o sea:

De las proposiciones con doble alternativa (si-no), a los operadores de proyección del espacio de Hilbert en que se representa el sistema.

(b) De la formulación matemática standard a la abstracción algebraico-conjuntista, o sea:

De los proyectores del espacio de Hilbert, al retículo ortomodular de proposiciones.

Finalmente, obtenemos el conocido esquema:

La estructura $(\mathrm{PR}(\mathrm{H}), \wedge, \mathrm{v}, ', \tilde{0}, \mathrm{i})$ es un modelo de los axiomas de la teoría de retículos (o conjuntos parcialmente ordenados) ortomodulares, donde $a \wedge b$ es la intersección de los subespacios cerrados $a$ y $b$ (asociados con los proyectores), $a$ v $b$ es la cápsula lineal de la unión de los subespacios, y $a^{\prime}$ es el espacio ortogonal con respecto a la forma hermítica que define el producto escalar en $\mathrm{H}$.

Lo que es menos conocido, y ha sido muy poco estudiado hasta ahora, es el grado de "fuerza" lógica que deben tener las teorías utilizadas para formalizar ese esquema. Por ejemplo, si planteamos la construcción de un universo para la mecánica cuántica en términos de la teoría axiomática de 
conjuntos, tal vez podríamos apreciar cuál es el "monto" mínimo de lógica necesario para la fundamentación de la matemática cuántica.

Que yo sepa, el primer esfuerzo coherente hecho en ese sentido, es el excepcional trabajo de Benioff [1] y [2]. En [1], Benioff se plantea seriamente el problema de considerar cuáles son las (eventuales) variaciones sufridas por una teoría física, cuando se eligen universos diferentes (en el sentido de la teoría axiomática de conjuntos, por ejemplo, $\mathrm{ZF}$ ), como portadores de la matemática necesaria para la física. En particular, el autor prueba (p. 626), que el modelo mínimo $\mathrm{M}_{0}$ de la teoría de Zermelo Fraenkel (con axioma de elección), no es adecuado para la expresión de la noción fuerte de aleatoricidad. En [2], p. 637, esta limitación de ciertos modelos para servir como portadores de la matemática cuántica es extendida a otras estructuras de la teoría de conjuntos.

Esos resultados, además de sus poderosas implicaciones técnicas, tanto en el campo de la fundamentación de la física, como en la metamatemática misma, aportan también reflexiones metodológicas muy interesantes, por ejemplo, sobre la relevancia del tipo de lógica a usar.

En efecto, aunque con antecedencia a los trabajos de Benioff, pocas veces fue contemplada la posibilidad de elegir universos matemáticos para la física que fueran más pequeños que la clase universal $V$ de Cantor (es decir, la clase universal restringida a su parte formalizable en $\mathrm{ZF}$ ), en algunos casos se acarició la esperanza de que la matemática necesaria para la ciencia empírica fuera relativamente "modesta" y pudiera, por lo tanto, ser susceptible de formalizaciones más débiles que las que requieren la plena potencia de la lógica clásica. Pero los resultados de Benioff muestran que esos universos tampoco pueden ser "demasiado pequeños", razón ésta que hace sospechar que sus respectivas metateorías no pueden ser demasiado débiles.

Podría argumentarse, sin embargo, de la siguiente mane- 
ra: la tarea de la lógica no es fabricar modelos de teorías, sino simplemente dar reglas para hacer deducciones dentro de teorías. En todo caso, la construcción de modelos es una tarea de la metamatemática, que a su vez usa una lógica clásica. De cualquier manera, el problema pendiente es el de determinar cuál es el mínimo de lógica que necesitamos para desenvolver toda la matemática que deseamos interpretar en esos universos. Es bastante difícil de cuestionar que en el desenvolvimiento de esas teorías, los teoremas de la lógica clásica parecen todos necesarios. En todo caso, deberíamos esperar por una versión cuántica de la lógica, análoga a la versión intuicionista, que permitiera desarrollar una matemática compatible con ella. Y habría que desear, en ese caso, que su éxito fuera un poco mayor que el de ésta.

\section{Conclusiones}

Como hemos visto en el parágrafo 1 , la formulación ortodoxa de la "lógica" cuántica consiste en la construcción de una estructura algebraica. Podemos considerar dos variantes: un retículo ortomodular y atómico $\mathrm{L}$, o un par ordenado $(L, M)$ en donde $L$ es un conjunto parcialmente ordenado y ortomodular, y $\mathrm{M}$ un conjunto de funciones sigma-aditivas, que separan los puntos de L, y que constituyen medidas de probabilidad sobre L. Otras formulaciones intermedias son posibles.

Desde este punto de vista está claro que:

(1) Las estructuras así construidas no son sistemas de lógica.

En efecto: este "truismo" se verifica por simple inspección. Un sistema de lógica, sea clásica o no, es un lenguaje formado por variables y constantes, más un sistema formal constituido por axiomas puramente lógicos y reglas de inferencia. Las lógicas cuánticas pueden considerarse entonces, álgebras asociadas a ciertos sistemas de lógica proposicional no clásica. 
(2) Dichas estructuras pueden ser consideradas también como modelos de ciertas teorias.

Pero esas teorías tienen como lógica subyacente la lógica de cuantificadores clásica. Observemos, por ejemplo, el comportamiento del especialista en teoría de modelos o en lógica algebraica, cuando manipula los axiomas de una teoría muy débil, como lo es la de retículos ortomodulares. El especialista sabe que si tiene el polinomio

$$
x \wedge(y \vee z)
$$

no puede sustituirlo por su "colega": $(x \wedge y)$ v $(x \wedge z)$, a menos que quiera incurrir en serias incoherencias formales, ya que ambos no proporcionarán las mismas funciones en L.

A pesar de eso, si él ha conseguido demostrar que:

(十) " $a$ es un complemento de $b$ en L"

y, también, ha podido probar que:

$(++)$ "el único complemento de $a$ es $b$ " (

o bien "existen varios complementos de $b$ " (=q)

entonces, él usará sin ningún problema, la proposición

$$
((+) \text { y } \mathbf{p}) \text { o }((+) \text { y q })
$$

sin dudar de que ella es consecuencia lógica de $(+)$ en conjunción con $(t+)$.

Analogías mucho más burdas pueden mostrar lo obvio de nuestra observación. Si $E$ es una estructura con una relación binaria irreflexiva $R$, y tenemos un lenguaje con un solo símbolo $R^{\prime}$, entonces, está claro que $R^{\prime}(x, x)$ es falsa en $E$. Sin embargo, el lógico no tendría derecho a considerar que en la lógica en la cual desenvuelve su teoría, hay alguna relación privilegiada que puede ser "asemejada" con $R^{\prime}$.

Lo mismo sucede al comparar la teoría de retículos con la lógica misma. El supremo e ínfimo de proposiciones cuánticas, tiene fuertes analogías con sus equivalentes de la lógica, pero no tenemos por qué considerar a ellos como la 
versión algebraica de la disjunción y conjunción usuales. Sin embargo, ellos tienen cierto significado lógico en el sentido afirmado por la siguiente "tesis":

(3) Las proposiciones que son elementos de una estructura cuántica describen hechos empíricos.

En efecto, si consideramos cualquiera de las experiencias más simples de la mecánica cuántica clásica, como, por ejemplo, la incidencia de un haz de partículas sobre una pantalla provista de un pequeño orificio, a través del cual el haz se dispersa, proyectando en una placa fotográfica, las proposiciones que naturalmente se presentan son del tipo siguiente: "la partícula $e$ está en el punto $x_{1}$ " (en un momento dado)

Si tenemos tres proposiciones $a, b$ y $c$, puede naturalmente suceder que el operador de proyección asociado con

$$
a \wedge(b \vee c)
$$

no coincida con el que corresponde a

$$
(a \wedge b) \vee(a \wedge c)
$$

Eso significa simplemente que la terna $(a, b, c)$ no es compatible, o, a lo sumo, que las dos proposiciones no pueden ser reemplazadas mutuamente como objetos de $\mathrm{L}$, pero no que la ley lógica de distributividad no sea una regla válida, ya que $L$ es un retículo, no una lógica, y por tanto carece de sentido hablar de reglas.

Habríamos probado que realmente se necesita una lógica especial en la mecánica cuántica, si la teoría de operadores lineales en el espacio de Hilbert utilizara, para las inferencias matemáticas habituales, reglas o axiomas no clásicos. Más aún, deberíamos probar que el uso de la ley distributiva a ese nivel, $\mathrm{A} \&(\mathrm{~B}$ v C) $\vdash(\mathrm{A} \& \mathrm{~B}) \mathrm{v}(\mathrm{A} \& \mathrm{C})$, conduce $a$ probar teoremas que contrarian nuestra experiencia física. Lo que en realidad sucede es que, en el retículo, no se verifica la desigualdad entre los polinomios (i) y (ii). 
En realidad, para que el enunciado (3) pierda un poco su carácter demasiado trivial, debería ser reformulado ahora de la siguiente manera:

$\left(3^{\prime}\right)$ Las operaciones entre proposiciones que son elementos de una estructura cuántica, describen situaciones puramente empíricas (como posibilidad de medición simultánea, compatibilidad de ciertas observaciones, etc.).

En el parágrafo 2, observamos, muy estrechamente en concordancia con van Fraassen, que las construcciones cuánticas desempeñan un papel importante en las formulaciones habituales del modelo empírico de la teoría cuántica. $O$ sea, existe un cierto nivel empírico en el cual se dan los "hechos de la física" (cual sea el status de ese nivel es un problema filosófico que cae fuera del campo de la epistemología); las relaciones en ese nivel, son conceptualizadas por construcciones formales, que intentan reproducir, en el plano semántico, las situaciones fácticas. Esas construcciones (retículos, álgebras) son susceptibles de análisis con los métodos matemáticos usuales, y, eventualmente, pueden ser estudiadas por la teoría de modelos. Finalmente, su "esencia" puede ser captada por sistemas proposicionales de los cuales ellas constituyen la versión algebraica.

La relación entre las estructuras cuánticas, y la lógica proposicional, es, como siempre, doble: las estructuras dan sentido al cálculo de proposiciones, y éste desarrolla mecánicamente, a través de sus reglas, los teoremas que "hablan" de la estructura. Hay, sin embargo, un punto importante: el análisis formal de las estructuras no precisa de una lógica proposicional especial. Esa lógica es un objeto ad hoc construido por los lógicos no clásicos por interés intrínseco. El formalismo de la teoría de retículos, que se basa a su vez en la lógica clásica, sería suficiente. Luego:

(4) La construcción de una lógica proposicional cuântica, cuya álgebra asociada sea una estructura cuántica, no aporta resultados nuevos sobre el conocimiento de dicha estructura. Eventualmente, la comparación entre la lógica y la estructu- 
ra puede ser útil para la lógica misma (p.ej., para decidir ciertos problemas metalógicos).

Abordemos finalmente el problema crucial: $¿$ No hará falta una lógica que sirva como base para las teorías matemáticas, las cuales, a su vez, darán cuenta de los resultados de la mecánica cuántica?

Pensemos en la construcción de Benioff. El universo matemático que constituye un portador adecuado para la mecánica cuántica tal vez no precise ser el universo $V$, pero indudablemente, tampoco es un modelo minúsculo. La matemática que podemos desarrollar dentro de él, y que, sin duda, deberemos desarrollar, no puede ser muy pequeña. Ahora bien: podría pensarse, sin embargo, que eso no establece limitaciones muy serias con respecto a la lógica. Podríamos construir lógicas más débiles que la clásica pero que permitan la deducción de todos los teoremas necesarios para la matemática e inclusive "muchos" más.

Esa posibilidad, empero, no está lejos de ser una idealización innecesaria. Con el mismo criterio, podría pensarse en una lógica "menor" que la clásica, apta para la totalidad de la matemática. Algunas veces se ha pretendido que en el razonamiento habitual en matemática no se usan las leyes lógicas en su totalidad, y tal vez nos podríamos reducir a las estrictamente necesarias. Es dudoso, en nuestra opinión, que si adoptamos esas leyes, no obtengamos finalmente, como "clausura deductiva" de las mismas, la lógica usual.

Por otro lado, la existencia de una lógica "menor" que cumpla la misma función, no parece un justificativo suficiente para abandonar la clásica, excepto por el célebre requisito de economía, que a menudo implica serias distorsiones conceptuales. Por ejemplo, podemos desarrollar toda la topología con un único y complicadísimo postulado, pero ¿cuál es la ventaja de hacer eso, cuando tenemos formulaciones "naturales" con más axiomas, que presentan conceptos tan claros como el de abierto, interior, u operador de clausura? 
Una razón auténticamente válida para la sustitución de la lógica sería la de construir una teoría cuántica especial, basada en una matemática, también especial, en que ciertos teoremas clásicos ya no valdrían, así como en la matemática intuicionista no valen los teoremas clásicos que son equivalentes al axioma de elección. $O$ sea, no bastaría decir que con la nueva lógica se puede probar todo lo "necesario" que era demostrable con la lógica clásica, sino también que con ella no se pueden probar ciertas proposiciones "objetables" que en la lógica clásica eran teoremas. Resultaría entonces que habría una nueva mecánica, cuya plausibilidad empírica tal vez diera mucho que pensar.

De hecho, las lógicas no clásicas (con la posible excepción del intuicionismo y de la modal) tienen un interés exclusivamente filosófico, o, mejor aún, lógico, en el sentido más "intrínseco" posible de la palabra. Pero entonces será un poco aventurado buscarle justificaciones a través del trabajo real de la ciencia. A menos que modifiquemos axiomáticamente también la realidad, lo cual no debe estar muy lejos de las ambiciones de muchos epistemólogos y lógicos "heterodoxos".

\section{REFERENCIAS}

[1] Benioff, P. A. Journal of Mathematical Physics, 17, p. 618.

[2] Benioff, P. A. Journal of Mathematical Physics, 17, p. 629.

[3] Bunge, M. American Journal of Physics, 24, p. 272.

[4] Bunge, M. Foundations of Physics. New York: Springer, 1967.

[5] Bunge, M. (ed.). Quantum Theory and Reality. New York: Springer, 1967.

[6] Deliyannis, P. C. Journal of Mathematical Physics, 17, p. 248.

[7] Gudder, S. P. Pacific Journal of Mathematics, 19, p. 81.

[8] Gudder, S. P. Journal of Mathematical Physics, 11, p. 1037.

[9] Hardegree, G. M. Z. Naturforsch, 30 a, p. 1347.

[10] Jauch, J. Foundations of Quantum Theory. New York: Addison-Wesley, 1968.

[11] Kochen, S. \& Specker, E. Symposium on the Theory of Models. NorthHolland, 1965, p. 177.

[12] Kotas, J. Studia Logica, XXI, p. 17.

[13] Kotas, J. Studia Logica, XXVII, p. 73.

[14] Merzbaacher, E. Quantum Mechanics. New York: Wiley, 1970.

[15] Piron, C. Helv. Phys. Acta 37, p. 439.

[16] Pulmannova, S. Comm. in Mathematical Physics, 49, p. 47. 
[17] Raziowa, H. An Algebraic Approach to Non-Classical Logics. Amsterdam: North-Holland, 1974.

[18] Shoenfield, J. Mathematical Logic. New York: Addison-Wesley, 1967.

[19] Van Fraassen, en Hooker. Contemporary Research in the Foundations and Philosophy of Quantum Theory. Dordrecht: Reidel, 1973.

[20] Van Fraassen, en Hooker. The LogicaAlgebraic Approach to Quantum Mechanics. Dordrecht: Reidel, 1975. 
The purpose of this paper is to prove that the construction of a quantum logic is a task of specific interest for non-classical logic, but that it is relevant neither to the methodology of quantum mechanics nor to the formalization of the alternative yes-no process. The main argument is that quantum structures (for example, ortho. complemented partially ordered sets, orthomodular lattices and partial algebras) are not systems of logic proper, but rather are perfectly formalizable structures in classical logic.

In $\S 1$, the orthodox formulation of Jauch and Piron are analyzed. I affirm that the objections made by these authors against "quantum logic" are not eliminated by the introduction of a conditional in the lattice structures, since the true reason why quantum "logic" is not really logic lies in its formulation, which is not that of a propositional system, but rather that of a specific theory whose model is these lattices.

It is argued that the collection of the properties satisfied by certain objects of a structure does not justify their presentation as purely logical properties, nor does the presence of certain operations of the least upper bound and greatest lower bound permit us to call symbols introduced by analogy in propositional logic "connectives". In any case, if that formalization is indeed possible, it does not imply the adoption of a new logic as a formal instrument for quantum mechanics.

In $\S 2$, aspects of quantum theories which are especially semantic in nature are analyzed. I agree with van Fraassen's statement in the sense that that semantic formulation makes specific concepts of the theory clearer, but it does not attempt to construct its own logic. I deduce that it is non-classical logic which can actually take advantage of quantum semantics, as a model which, eventually, could throw light on the metalogical properties of certain special propositional systems.

Finally, by using Benioff's profound set theory analysis, I advance the hypothesis that classical logic is possibly the only one that can account for the richness of the meanings that appear in quantum mechanics. 